\title{
Hereditary bilateral sudden sensorineural hearing loss
}

\author{
$\operatorname{Varga} \mathrm{L}^{1,2}$, Jovankovicova $\mathrm{A}^{3}$, Huckova $\mathrm{M}^{2}$, Demesova $\mathrm{L}^{1,2}$, Gasperikova $\mathrm{D}^{2}$, Sebova $\mathrm{I}^{3}$, Profant $\mathrm{M}^{1}$ \\ Department of Otorhinolaryngology - Head and Neck Surgery, Faculty of Medicine and University Hospital, \\ Comenius University, Bratislava, Slovakia. profant@fnorl.sk
}

\section{ABSTRACT}

OBJECTIVES: The aim of our study is to demonstrate a causal link between two distinct diagnoses, the hereditary hearing loss, and the sudden sensorineural hearing loss.

BACKGROUND: Sudden sensorineural hearing loss is an emergency condition in otolaryngology and a rare diagnosis in childhood. Most often it only affects one ear and its cause remains unknown.

METHODS: We present a clinical study of a 10 -year-old female patient presenting with bilateral sudden sensorineural hearing loss analyzed by Sanger sequencing of the GJB2 gene.

RESULTS: The subject was referred to the hospital for bilateral sudden hearing loss which developed 3 days before the admission. Audiometric testing confirmed bilateral asymmetric sensorineural hearing loss. All routine diagnostic procedures including MRI and CT imaging showed normal results. She was treated with intravenous and intratympanic corticosteroids followed by hyperbaric oxygen therapy with partial hearing recovery in one ear. DNA analysis of the GJB2 gene identified biallelic c.35delG deletion. The subject had no other affected family members and her auditory development to that time was normal.

CONCLUSION: Our finding extends the knowledge on phenotype variability in GJB2 variants. We suggest considering genetic testing in pediatric cases of bilateral sudden sensorineural hearing loss (Tab. 1, Fig. 4, Ref. 24). Text in PDF www.elis.sk.

KEY WORDS: sudden hearing loss, bilateral, DNA analysis, connexin 26, GJB2.

\section{Introduction}

Sudden sensorineural hearing loss (SSNHL) is characterized by rapid onset (within 72 hours) affecting at least three consecutive frequencies with hearing thresholds of $\geq 30 \mathrm{~dB}$. It is traditionally considered as an emergency condition in otolaryngology and early treatment is known to provide higher rate of hearing recovery (1). According to more recent studies the incidence of SSNHL in adults ranges between 10.2-27: $100000(2,3)$. In the vast majority of the cases $(95-99 \%)$ it only affects one ear, while bilateral cases are rare $(1-5 \%)(4,5)$. Moreover, bilateral SSNHL is associated with more severe underlying diagnoses, poorer prognosis and higher mortality (6). The epidemiology of SSNHL in children is unknown as most of the available data are based on rather small

${ }^{1}$ Department of Otorhinolaryngology - Head and Neck Surgery, Faculty of Medicine and University Hospital, Comenius University, Bratislava, Slovakia, ${ }^{2}$ Diabgene Laboratory, Biomedical Research Center, Slovak Academy of Sciences, Bratislava, Slovakia, and ${ }^{3}$ Pediatric ORL Department, Faculty of Medicine and National Institute of Children's Diseases (NICD), Comenius University, Bratislava, Slovakia

Address for correspondence: M. Profant, MD, PhD, Department of Otorhinolaryngology - Head and Neck Surgery, Faculty of Medicine and University Hospital, Comenius University, Antolska 11, SK-851 07 Bratislava, Slovakia.

Phone: +421-2-68672052

Acknowledgement: This work was supported by grants APVV 15-0067, VEGA 1/0214/16 and ITMS 26240220087. case series. Nevertheless, it seems that young children with SSNHL have a poorer prognosis than adolescents $(7,8)$.

\section{Clinical study}

A 10-year-old female patient was referred to the Pediatric ENT department at NICD in Bratislava in February 2019 with 3-day clinical history of SSNHL in both ears. About one week prior to hearing loss onset, she complained of sore throat and was prescribed Penicillin for streptococcal tonsillitis based on positive bacterial culture. The subject was otherwise healthy, had normal

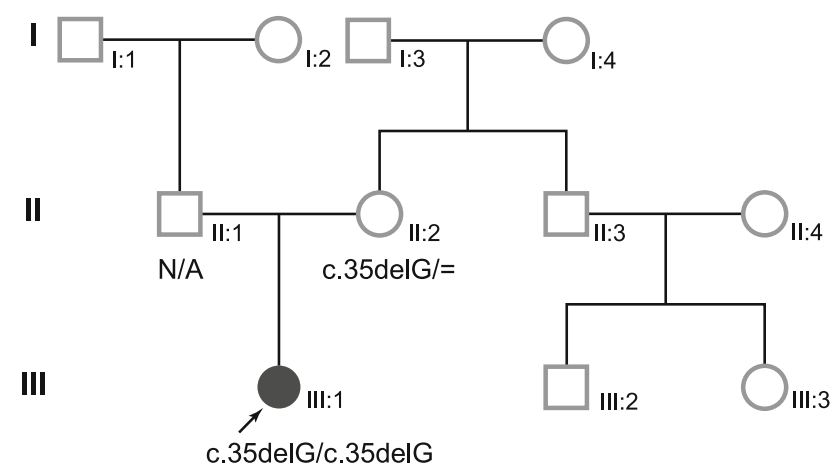

Fig. 1. Pedigree of the proband. Filled symbol- affected individual, empty symbol - normal hearing individual, arrow-proband. 

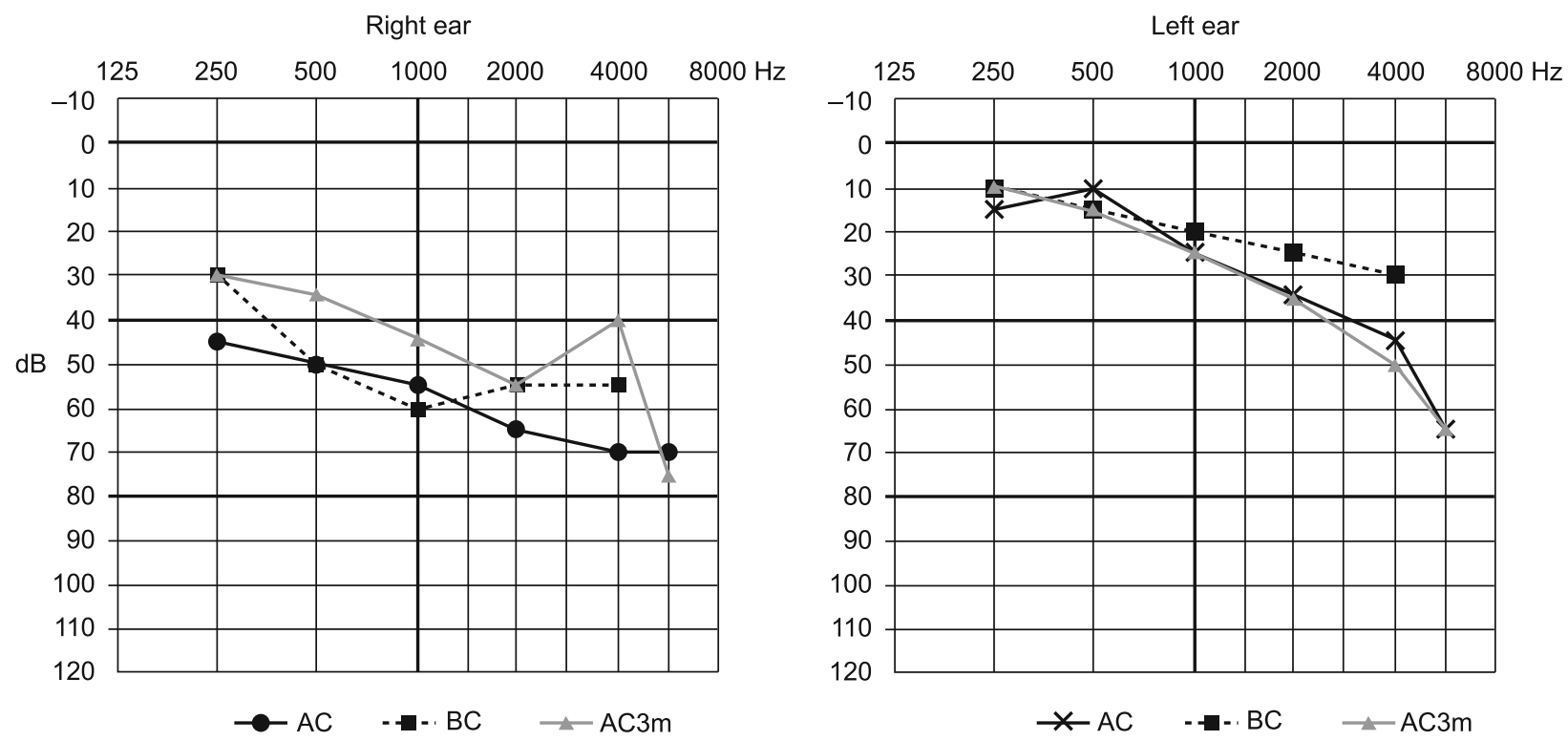

Fig. 2. Pure tone audiogram of the proband showing asymmetric hearing loss (left ear - mild degree, right ear - moderate degree). Colored full lines - initial air conduction thresholds, colored dashed lines - initial bone conduction thresholds, gray lines - air conduction thresholds after three-month follow-up.

language development (bilingual) and attended regular school without learning problems or any other signs suggesting previous hearing loss. Family history of hearing loss was negative (Fig. 1). Physical ENT examination showed normal findings. Audiological evaluation confirmed asymmetric bilateral sensorineural hearing loss (Fig. 2) with absent otoacoustic emissions (DPOAE), normal auditory brainstem response (ABR) latencies, A-type tympanometric curves, and positive recruitment based on acoustic reflex thresholds indicating cochlear damage. Pure tone average $(0.5$, $1,2,4 \mathrm{kHz}$ ) thresholds were $28.75 \mathrm{~dB}$ for the left ear and $60 \mathrm{~dB}$ for the right ear. The subject did not complain of tinnitus or dizziness and basic vestibular investigation demonstrated normal vestibular functions.

The subject was admitted for intravenous therapy and further investigations. All laboratory tests (complete blood count, coagulation, serum biochemistry, urinalysis, serology for CMV and EBV) showed normal results with exception of positive anti-EBV IgG
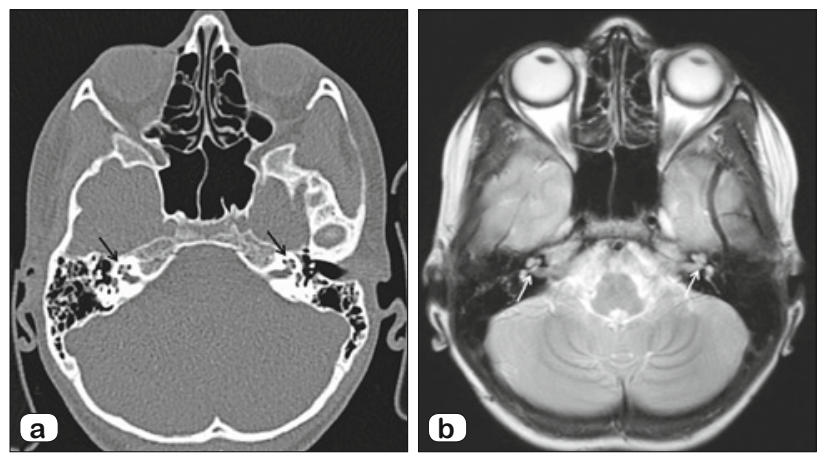

Fig. 3. Brain and temporal bone CT (a) and T2 weighted MRI (b) scans in axial plane demonstrating normal inner ear anatomy (arrows). antibodies. Ophthalmologist diagnosed mild hypermetropia, but apart from that, the subject had normal retinal findings. Neurological investigation did not reveal any focal neurological signs. Both CT and MRI of the brain and temporal bone showed normal anatomy of the inner ear and pontocerebellar angle (Fig. 3). Based on routine diagnostic protocol the subject was further treated as idiopathic SSNHL case. Systemic corticosteroids (methylprednisolone) were administered intravenously in a taper dose over 14 days. Because no hearing recovery was observed within 5 initial days after hospital admission the treatment was boosted with 3 consecutive shots of intratympanic methylprednisolone in both ears under propofol anesthesia. Moreover, the patient underwent 20 sessions of hyperbaric oxygen therapy after hospital discharge. Three months after hearing loss onset and treatment the audiogram showed a slight improvement of thresholds in the right ear, while it remained unchanged in the left ear (Fig. 2).

However, since bilateral SSNHL cases are rare, peripheral blood and buccal swab were also taken during hospital stay for DNA analysis of hereditary hearing loss despite negative family history and postlingual onset of hearing loss. The non-coding (exon 1) and coding region (exon 2) of the GJB2 gene were amplified and analyzed by Sanger sequencing as the first step in our otogenetic diagnostic pipeline. Surprisingly, the subject turned out to be a homozygous carrier of c.35delG deletion (Fig. 4), which was confirmed by repeated analysis of two independent samples. The only other family member available for genetic testing was her mother who was detected to be a c.35delG heterozygous carrier. We further retrospectively analyzed the phenotypes in other 163 individuals with biallelic c.35delG variant from our DNA repository of sensorineural hearing loss, but we did not find any other subject with sudden postlingual onset of hearing loss. 
c c t g g g g g g t $\underline{g ~ t ~ g ~ a ~}$

. . . . . . . . . . . .

C C T G G G G G G T G T G A reference sequence

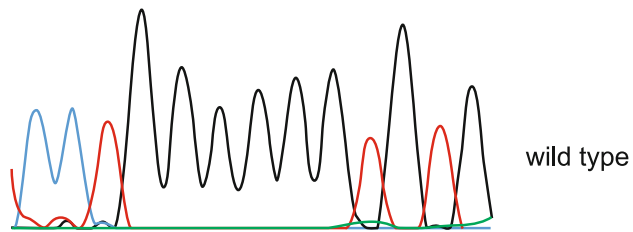

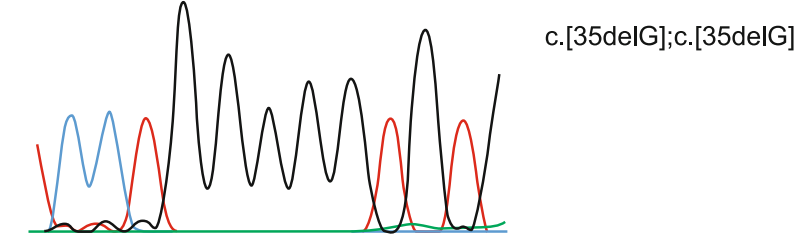

Fig. 4. Sanger sequencing electropherogram of the proband showing homozygous c.35delG deletion compared to wild type. The deletion of one guanine nucleotide is depicted by the arrow.

\section{Discussion}

Despite adequate clinical investigation, distinct etiological diagnosis could only be established in about $10-30 \%$ of SSNHL cases. The remaining majority is regarded as idiopathic. The most prevalent known causes of this heterogenic disease include infectious and ear diseases, trauma, vascular or hematologic disorders, tumors, ototoxic agents and autoimmune diseases $(1,9)$. In pediatric population the two most frequently identified etiologies involved infectious (viral) causes and inner ear malformations, particularly the enlarged vestibular aqueduct (EVA) (10-12). The subject presented in this report did not show any signs of acute infection at the time of hospital admission although an episode of acute tonsillitis few days before the hearing loss onset was noted in her clinical files. Similarly, the imaging studies (CT and MRI) excluded any inner ear or intracranial malformations.

The possible role of genetic factors in SSNHL had been largely overlooked in the past, despite sporadic records of familial SSNHL cases (13). In general, genes and variants with autosomal recessive inheritance cause congenital or early onset (prelingual) deafness, whereas genes with autosomal dominant inheritance lead to progressive hearing loss often with delayed onset. However, about one-half of patients with bilateral and about $10 \%$ with unilateral EVA harbor biallelic SLC26A4 mutations associated with autosomal recessive nonsyndromic deafness or Pendred syndrome (14, $15)$. These patients may suffer from sudden hearing loss even after minor head trauma (16). This is in contrast with GJB2 variants, which are typically associated with nonsyndromic autosomal recessive bilateral sensorineural hearing loss with congenital or prelingual onset. The degree of hearing loss in GJB2 related deafness is variable and depends on specific genotype. In c.35delG deletion, the most frequent pathogenic GJB2 variant in Caucasians, which was also detected in the presented subject, about $90 \%$ of homozygotes demonstrate early onset severe to profound deafness (17).

Reports on episodes of apparently sudden hearing loss in biallelic GJB2 mutations are scarce with only 6 cases described in the literature (18-21) (Tab. 1). However, half of them were observations from deaf families with several affected family members where genetic etiology of hearing loss could be expected. With one exception (19) they also occurred in association with those GJB2 variants which generally cause hearing loss of mild to moderate degree, such as c. $269 \mathrm{~T}>\mathrm{C}, \mathrm{c} .235 \mathrm{delC}$ and c. $109 \mathrm{G}>\mathrm{A}$. Additionally, patients homozygous or compound heterozygous for the variant c. $109 \mathrm{G}>$ A have often delayed hearing loss onset and rarely they may even have normal hearing, which led to past concerns regarding its pathogenicity $(22,23)$. Moreover, SSNHL episodes in previously published cases did not occur in normal hearing individuals, but as a rapid worsening of previously diagnosed hearing loss. Based on normal auditory behavior in the past, there was no suspicion of hearing loss in the presented subject and thus premorbid audiogram was not available. Similarly, no data are available from neonatal hearing screening (NHS), because she was born in another country, where universal NHS has not yet been established. However, it is assumed that GJB2 associated deafness is not always congenital and that an early window of functional hearing may exist in some cases before its final deterioration (24). This is also supported by several own observations of children who initially passed NHS and were diagnosed with GJB2 related deafness later during the

Tab. 1. Causative GJB2 genotypes detected in association with SSNHL.

\begin{tabular}{|c|c|c|c|c|c|c|}
\hline $\begin{array}{l}\text { Case } \\
\text { number }\end{array}$ & Genotype & $\begin{array}{l}\text { Laterality } \\
\text { of SSNHL }\end{array}$ & $\begin{array}{l}\text { Age at SSNHL } \\
\text { episode }\end{array}$ & $\begin{array}{l}\text { Known HL prior } \\
\text { to SSNHL episode }\end{array}$ & $\begin{array}{c}\text { Family history } \\
\text { of HL }\end{array}$ & Resource \\
\hline 1 & c. $35 \mathrm{delG} / \mathrm{c} .269 \mathrm{~T}>\mathrm{C}$ & N/A & adolescence & yes & familial & Janecke et al (18) \\
\hline 2 & c. $35 \mathrm{delG} / \mathrm{c} .269 \mathrm{~T}>\mathrm{C}$ & unilateral & 13 years & yes & familial & Janecke et al (18) \\
\hline 3 & c. $35 \mathrm{delG} / \mathrm{c} .35 \mathrm{delG}$ & unilateral & 23 years & yes & familial & Kokotas et al (19) \\
\hline 4 & c. $109 \mathrm{G}>\mathrm{A} / \mathrm{c} .109 \mathrm{G}>\mathrm{A}$ & simultaneous bilateral & 6 years & N/A & sporadic & Chen et al (20) \\
\hline 5 & c. $109 \mathrm{G}>\mathrm{A} / \mathrm{c} .109 \mathrm{G}>\mathrm{A}$ & unilateral & 41 years & yes & sporadic & Chen et al (20) \\
\hline 6 & 235delC/ 235delC & unilateral & N/A & yes & sporadic & Wang et al (21) \\
\hline
\end{tabular}


prelingual period (unpublished data). Thus the presented case may represent an example of extremely extended functional window. Her clinical manifestation clearly fulfilled the diagnostic criteria for SSNHL and without genetic testing, it would be classified into the idiopathic subgroup. Mechanisms leading to these extreme but also rare phenotypes are not yet fully understood. It is hypothesized that overexpression of GJB6 (connexin 30) or presence of modifier genes may at least partially explain this phenomenon (21).

\section{Conclusion}

SSNHL is a rare condition in pediatric population. Bilateral cases may be regarded as extremely rare and require extensive diagnostic workup as they may represent the first symptom of potentially life-threatening underlying disease. We provide further evidence, that SSNHL may be associated with genetic etiology and that genetic testing should be considered in diagnostic protocol for bilateral SSNHL. Moreover, this is the first report of simultaneous bilateral SSNHL in postlingual period in a presumably normal hearing subject homozygous for c.35delG deletion in GJB2 gene which extends the knowledge on phenotypes in this most frequently affected deafness gene.

\section{References}

1. Stachler RJ, Chandrasekhar SS, Archer SM et al. Clinical practice guideline: sudden hearing loss. Otolaryngol Head Neck Surg 2012; 146 (Suppl 3): S1-35.

2. Wu CS, Lin HC, Chao PZ. Sudden sensorineural hearing loss: evidence from Taiwan. Audiol Neurootol 2006; 11 (3): 151-156.

3. Alexander TH, Harris JP. Incidence of sudden sensorineural hearing loss. Otol Neurotol 2013; 34 (9): 1586-1589.

4. Oh JH, Park K, Lee SJ, Shin YR, Choung YH. Bilateral versus unilateral sudden sensorineural hearing loss. Otolaryngol Head Neck Surg 2007; 136 (1): 87-91.

5. Kitoh R, Nishio SY, Ogawa K et al. Nationwide epidemiological survey of idiopathic sudden sensorineural hearing loss in Japan. Acta Otolaryngol 2017; 137 (Suppl 565): S8-S16.

6. Chen YH, Young YH. Bilateral simultaneous sudden sensorineural hearing loss. J Neurol Sci 2016; 362: 139-143.

7. Kim JY, Han JJ, Sunwoo WS et al. Sudden sensorineural hearing loss in children and adolescents: Clinical characteristics and age-related prognosis. Auris Nasus Larynx 2018; 45 (3): 447-455.

8. Chen K, Jiang H, Zong L, Wu X. Side-related differences in sudden sensorineural hearing loss in children. Int J Pediatr Otorhinolaryngol 2018; 114: 5-8.

9. Chau JK, Lin JR, Atashband S, Irvine RA, Westerberg BD. Systematic review of the evidence for the etiology of adult sudden sensorineural hearing loss. Laryngoscope 2010; 120 (5): 1011-1021.
10. Tarshish Y, Leschinski A, Kenna M. Pediatric sudden sensorineural hearing loss: diagnosed causes and response to intervention. Int J Pediatr Otorhinolaryngol 2013; 77 (4): 553-559.

11. Dedhia K, Chi DH. Pediatric sudden sensorineural hearing loss: Etiology, diagnosis and treatment in 20 children. Int J Pediatr Otorhinolaryngol 2016; 88: 208-212.

12. Pitaro J, Bechor-Fellner A, Gavriel H, Marom T, Eviatar E. Sudden sensorineural hearing loss in children: Etiology, management, and outcome. Int J Pediatr Otorhinolaryngol 2016; 82: 34-37.

13. Gäckler A, Eickelmann AK, Brors D, Dazert S, Epplen JT, Kunstmann E. Positive family history of idiopathic sudden sensorineural hearing loss. Eur Arch Otorhinolaryngol 2010; 267 (12): 1843-1848.

14. Chattaraj P, Reimold FR, Muskett JA et al. Use of SLC26A4 mutation testing for unilateral enlargement of the vestibular aqueduct. JAMA Otolaryngol Head Neck Surg 2013; 139 (9): 907-913.

15. Rah YC, Kim AR, Koo JW, Lee JH, Oh SH, Choi BY. Audiologic presentation of enlargement of the vestibular aqueduct according to the SLC26A4 genotypes. Laryngoscope 2015; 125 (6): E216-222.

16. Noordman BJ, van Beeck Calkoen E, Witte B, Goverts T, Hensen E, Merkus P. Prognostic factors for sudden drops in hearing level after minor head injury in patients with an enlarged vestibular aqueduct: a metaanalysis. Otol Neurotol 2015; 36 (1): 4-11.

17. Snoeckx RL, Huygen PL, Feldmann D et al. GJB2 mutations and degree of hearing loss: a multicenter study. Am J Hum Genet 2005; 77 (6): 945-957.

18. Janecke AR, Hirst-Stadlmann A, Günther B et al. Progressive hearing loss, and recurrent sudden sensorineural hearing loss associated with GJB2 mutations--phenotypic spectrum and frequencies of GJB2 mutations in Austria. Hum Genet 2002; 111 (2): 145-153.

19. Kokotas H, Theodosiou M, Korres $\mathbf{G}$ et al. Sudden hearing loss in a family with GJB2 related progressive deafness. Int J Pediatr Otorhinolaryngol 2008; 72 (11): 1735-1740.

20. Chen K, Sun L, Zong $\mathbf{L}$ et al. GJB2 and mitochondrial $12 \mathrm{~S}$ rRNA susceptibility mutations in sudden deafness. Eur Arch Otorhinolaryngol. 2016; 273 (6): 1393-1398

21. Wang HY, Xiong WP, Guan J, Wang QJ. A hereditary hearing loss case accompanied by unilateral sudden hearing loss: GJB2 235 delC homozygotes related late-onset moderate hearing impairment]. Lin Chung Er Bi Yan Hou Tou Jing Wai Ke Za Zhi 2016; 30 (14): 1136-1138.

22. Pollak A, Skórka A, Mueller-Malesińska M et al. M34T and V37I mutations in GJB2 associated hearing impairment: evidence for pathogenicity and reduced penetrance. Am J Med Genet A 2007; 143A (21): 2534-2543.

23. Shen $\mathbf{N}$, Peng $\mathbf{J}$, Wang $X$ et al. Association between the p.V37I variant of GJB2 and hearing loss: a pedigree and meta-analysis. Oncotarget 2017; 8 (28): 46681-46690.

24. Orzan E, Murgia A. Connexin 26 deafness is not always congenital. Int J Pediatr Otorhinolaryngol 2007; 71 (3): 501-507.

Received June 24, 2019. Accepted July 8, 2019. 